\title{
Switching from enoxaparin to dabigatran etexilate: pharmacokinetics, pharmacodynamics, and safety profile
}

\author{
Andreas Clemens • Joanne van Ryn • \\ Regina Sennewald • Norio Yamamura • \\ Joachim Stangier • Martin Feuring • Sebastian Härtter
}

Received: 18 October 2011 / Accepted: 22 December 2011 / Published online: 18 January 2012

(C) The Author(s) 2012. This article is published with open access at Springerlink.com

\begin{abstract}
Purpose Dabigatran etexilate is an oral, reversible, direct thrombin inhibitor licensed for the prevention of venous thromboembolism and stroke prevention in patients with atrial fibrillation. The aim of this study was to investigate whether, and to what extent, a switch from enoxparin to dabigatran etexilate affects the pharmacokinetic (PK) and pharmacodynamic (PD) parameters and safety profile of dabigatran.

Methods Enoxaparin $40 \mathrm{mg}$ was administered subcutaneously once daily for 3 days followed by a single dose of dabigatran etexilate $220 \mathrm{mg}$ (test treatment) on day 4 in an open-label, two-way cross-over trial in healthy volunteers. Dabigatran plasma levels were measured using a validated high-performance liquid chromatography tandem mass spectrometry method. Anticoagulant activity was measured using a number of clotting tests, including prothrombinase-
\end{abstract}

\footnotetext{
A. Clemens $(\bowtie) \cdot$ M. Feuring

Department of Global Clinical Development \& Medical Affairs,

Boehringer Ingelheim Pharma $\mathrm{GmbH}$,

Ingelheim am Rhein, Germany

e-mail: andreas.clemens@boehringer-ingelheim.com

J. van Ryn

Department of CardioMetabolic Disease Research,

Boehringer Ingelheim Pharma GmbH,

Biberach, Germany

R. Sennewald $\cdot$ N. Yamamura $\cdot$ S. Härtter

Department of Translational Medicine,

Boehringer Ingelheim Pharma $\mathrm{GmbH}$,

Biberach, Germany

J. Stangier

Department of Drug Metabolism and Pharmacokinetics,

Boehringer Ingelheim Pharma GmbH \& Co KG,

Biberach, Germany
}

induced clotting time (PiCT), activated partial thromboplastin time (aPTT), ecarin clotting time (ECT), and diluted thrombin time (dTT).

Results PK, PD, and safety data were available for 23 subjects for each treatment. The adjusted geometric mean test/reference ratio of area under the concentration-time curve for total dabigatran was $84 \%(90 \%$ confidence interval $67.2-105.0 \%)$ and $86 \%(67.0-110.0 \%)$ for maximum plasma concentration. The PiCT test/reference ratio, which represents the activity of enoxaparin and dabigatran, was elevated by approximately $15 \%$ for peak maximum effect ratio to baseline and total area under the effect curve $\left(\mathrm{AUEC}_{0-48}\right)$ activity, suggesting that some anticoagulant activity of enoxaparin was still present. Enoxaparin pre-treatment increased the $\mathrm{AUEC}_{0-48}$ of activated partial thromboplastin time by approximately $14 \%$. All other dabigatran-related PD markers were unaffected. Tolerability was good, with only mild and reversible adverse events during the treatment.

Conclusion Prior administration of enoxaparin did not meaningfully affect the PK or PD properties of dabigatran, and the switch from enoxaparin to dabigatran etexilate was well tolerated among the study subjects. These data support the safety of switching patients from enoxaparin to dabigatran etexilate.

Keywords Enoxaparin · Dabigatran etexilate · Switch · Pharmacokinetics $\cdot$ pharmacodynamics

\section{Introduction}

The benefits of antithrombotic therapy for preventing and treating venous thromboembolism (VTE) are well documented, and guidelines for treatment and prophylaxis have been developed by the American College of Chest 
Physicians, European Society of Cardiology, and other organizations to optimize patient management [1-6]. Dabigatran etexilate is a new, reversible, and direct thrombin inhibitor that is administered orally as a pro-drug and rapidly converted to the active form, dabigatran.

Dabigatran etexilate has been evaluated in Phase III trials for a number of indications. It has been found to have potential advantages during prolonged oral prophylactic therapy without the requirement for anticoagulation monitoring. In addition, as dabigatran etexilate has a rapid onset of action and can be given at fixed doses, its use can simplify treatment paradigms. This drug is licensed in $>75$ countries for the prevention of VTE following elective total hip or knee replacement surgery, including European countries [7] and Canada [8]. Dabigatran etexilate is also being investigated for the treatment and secondary prevention of VTE. In terms of the prevention of stroke or systemic embolism in patients with atrial fibrillation, dabigatran has shown superior efficacy compared with warfarin, with an improved safety profile $[9,10]$, and its use is approved for this indication in many countries, including the USA, Canada, Japan, Australia, and the European Union [8, 11, 12].

The replacement of a parenteral anticoagulant by an oral one, such as dabigatran etexilate, can potentially result in an easier and more successful implementation of prolonged prophylaxis. Studying the pharmacokinetic (PK) and pharmacodynamic (PD) parameters of dabigatran following this switch will contribute to our knowledge of this drug and, consequently, to the determination of whether it is a safe and effective treatment option.

Dabigatran conjugated with activated glucuronic acid forms a pharmacologically equipotent glucuronide that represents approximately $20 \%$ of total dabigatran exposure in plasma [13]. Hence, the sum of both free and conjugated dabigatran (total dabigatran) represents the appropriate correlate with PD. PD and dabigatran concentrations are closely correlated. The objective of this study was to investigate whether, and to what extent, a change from multiple subcutaneous (s.c.) doses (steady-state conditions) of enoxaparin $40 \mathrm{mg}$ once daily to a single oral dose of dabigatran etexilate $220 \mathrm{mg}$ affects the PK/PD parameters of dabigatran.

\section{Methods}

Study design and treatments

This study was a single-center, open-label, two-way crossover trial conducted in healthy men and women. The aim was to investigate the influence of a change from multiple s.c. daily doses of $40 \mathrm{mg}$ enoxaparin to a single oral dose of $220 \mathrm{mg}$ dabigatran etexilate on the relative bioavailability and PD parameters of dabigatran etexilate. It was designed to include 24 healthy male and female subjects, with at least ten of each gender. The trial was carried out in accordance with the Declaration of Helsinki (1996 version) and the International Conference on Harmonization Harmonized Tripartite Guideline for Good Clinical Practice (GCP). The study was sponsored by Boehringer Ingelheim Pharma GmbH \& Co. KG, Biberach, Germany.

Dabigatran etexilate (Pradaxa) was supplied by Boehringer Ingelheim Pharma $\mathrm{GmbH} \& \mathrm{Co} . \mathrm{KG}$ and was administered as a salt of methanesulphonic acid. For enoxaparin, commercially available Clexane prefilled syringes (produced by Sanofi-Aventis Deutschland GmbH, Frankfurt am Main, Germany) were used.

Each subject was allocated to one of two possible treatment sequences and was to receive each of the following treatments in a cross-over fashion: (1) enoxaparin $40 \mathrm{mg}$ administered s.c. once daily for 3 days followed by a single dose of oral dabigatran etexilate $220 \mathrm{mg} 24 \mathrm{~h}$ later on day 4 (test treatment) or (2) a single dose of dabigatran etexilate $220 \mathrm{mg}$, without prior enoxaparin administration (reference treatment). Cross-over treatment periods were separated by a wash-out phase of 5 days.

The trial comprised four visits. A screening examination (visit 1) was performed within 21 days before the first administration of the drug. During visits 2 and 3, the subjects were treated with either three daily doses of enoxaparin (days $-3,-$ 2 , and -1 ), followed by a single dose of dabigatran etexilate (day 1), or a single dose of dabigatran etexilate alone in the morning (day 1). An end-of-study examination (visit 4) was performed within 7 days after completion of visit 3 .

For the dabigatran etexilate treatment, subjects were discharged from the trial site on day 2. On day 3, the subjects returned for PK/PD blood sampling and an assessment of tolerability. For the enoxaparin/dabigatran etexilate treatment, subjects came to the trial site on days $-3,-2$, and -1 and were administered enoxaparin s.c. followed by dabigatran etexilate $24 \mathrm{~h}$ later (on day 1). PK/PD blood sampling and an assessment of tolerability were undertaken on days 1 to 3 .

\section{Subjects}

Healthy male or female subjects [based upon complete medical history, physical examination, vital signs (blood pressure, pulse rate), 12-lead electrocardiogram (ECG), clinical and laboratory tests], aged $\geq 18$ and $\leq 55$ years with a body mass index (BMI) between $\geq 18.5$ and $\leq 29.9 \mathrm{~kg} / \mathrm{m}^{2}$ were included into the trial. Participants gave their written informed consent in accordance with GCP and local legislation.

The exclusion criteria included any of the following: gastrointestinal, hepatic, renal, respiratory, cardiovascular, metabolic, immunologic or hormonal disorders, history of bleeding, or intake of medication which might influence clotting. 
Study endpoints

The primary PK endpoints were area under the concentration-time curve $\left(\mathrm{AUC}_{0-\infty}\right)$ and maximum plasma concentration $\mathrm{C}_{\max }$ ) for free and total dabigatran. Primary $\mathrm{PD}$ endpoints were area under the effect curve $\left(\mathrm{AUEC}_{0-48}\right)$ and maximum effect ratio to baseline $\left(E R_{\max }\right)$ of the combined anticoagulation activities of dabigatran and/or enoxaparin determined by the prothrombinase induced clotting test (PiCT) assay, as this assay is sensitive to both factor Xa inhibition and thrombin inhibition.

Secondary endpoints included other PK and coagulation parameters: diluted thrombin time (dTT), measured by HEMOCLOT (HYPHEN Biomed, Neuville sur Oise, France), activated partial thromboplastin time (aPTT), and ecarin clotting time (ECT), for dabigatran, plus PiCT for enoxaparin. Relative bioavailability and relative PiCT of the test treatment were compared with the reference treatment. Safety and tolerability were also secondary endpoints in this trial.

\section{PK/PD determinations}

Blood samples for dabigatran PK measurements were collected at -73.0 (before enoxaparin administration) and $-1.0 \mathrm{~h}$ (before dabigatran etexilate administration) and at $0.5,1,1.5,2,3,4,6,8,12,24,32$, and $48 \mathrm{~h}$ after the administration of dabigatran etexilate. Plasma concentrations of free, non-conjugated dabigatran, total dabigatran [sum of free and conjugated dabigatran (measured after alkaline cleavage of conjugates)], and the metabolites were determined by a validated high-performance liquid chromatography tandem mass spectrometry method at AAI Pharma Deutschland GmbH \& Co. KG, Neu-Ulm, Germany.

Briefly, for the determination of free dabigatran, $50 \mu \mathrm{L}$ of plasma was diluted with $50 \mu \mathrm{L}$ of $0.2 \mathrm{M}$ ammonium formate buffer ( $\mathrm{pH} 3.5$ ), spiked with $40 \mu \mathrm{L}$ internal standard solution (100 ng/mL [ $\left.{ }^{13} \mathrm{C} 6\right]$-dabigatran), mixed, and centrifuged. For the determination of total dabigatran, $50 \mu \mathrm{L}$ of plasma was spiked with $40 \mu \mathrm{L}$ internal standard solution and mixed with $20 \mu \mathrm{L}$ of $0.2 \mathrm{M}$ sodium hydroxide. After $2 \mathrm{~h}$ of incubation at $37^{\circ} \mathrm{C}$, the samples were acidified with $30 \mu \mathrm{L}$ of $0.2 \mathrm{M}$ hydrogen chloride, mixed, and then centrifuged.

The calibration curves for free and total dabigatran covered a range from 1.00 to $400 \mathrm{ng} / \mathrm{mL}$ plasma in undiluted samples. The calibration curves were linear over the calibration range. The validation data demonstrated adequate selectivity, accuracy $(-4.54 \%$ at maximum) and precision [coefficient of variation $(\mathrm{CV})$ of $5.28 \%$ at maximum] of the assay.

The prolongation of clotting times was calculated for each standard or trial sample in each assay. For all PD measurements, dabigatran standards at concentrations within the range $0.47-472 \mathrm{ng} / \mathrm{mL}$ were used for quality control purposes. For all PD measurements, one dabigatran quality control sample was measured at the beginning, in the middle, and at the end of the test phase of a day for quality control purposes. The mean $\mathrm{CV}$ of each coagulation time parameter across batches of quality control samples was $<5 \%$. The analysis of the coagulation parameters PiCT, dTT, aPTT, and ECT was performed by biochemA GmbH, Riegel, Germany. The determination of all clotting times was performed using a Merlin MC10 PLUS coagulometer (serial nos. 06 C 10004 and 08 C 10031; MERLIN Medical, Lemgo, Germany). For PK/PD correlations, blood plasma concentrations and coagulation times were analyzed in parallel.

The Pefakit PiCT assay (Pentapharm, Basel, Switzerland) was used to measure both factor Xa inhibition and thrombin inhibition by either dabigatran and/or enoxaparin and was performed according to the manufacturer's instructions. Briefly, a platelet-poor plasma sample $(50 \mu \mathrm{L})$ was mixed with $50 \mu \mathrm{L}$ of R1 activator reagent containing phospholipids and Russel's viper venom (factor V activator). After an incubation period $\left(3 \mathrm{~min}\right.$ at $37^{\circ} \mathrm{C}$ ), the added factor $\mathrm{Xa}$ is inhibited depending on the amount of antithrombin-heparin complexes and/or direct factor Xa and/or factor IIa inhibitors present in the sample. The measurement is then initiated by the addition of R2 Start Reagent $(50 \mu \mathrm{L} ; 25 \mathrm{mM}$ $\mathrm{CaCl}_{2}$ ). Following recalcification, the prothrombinase complex is formed using the residual factor Xa, phospholipids, factor Va generated from factor $\mathrm{V}$ present in the sample, and free calcium ions. Due to preactivation by factor V, prothrombinase formation is not dependent on endogenous thrombin-mediated factor $\mathrm{V}$ activation, resulting in this assay being named the "Prothrombinase-induced Clotting Time" (PiCT) assay. The time lag between the addition of $\mathrm{CaCl}_{2}$ and clot formation is detected in seconds as the PiCT, and we used these data in the final presentation of the data.

The dTT was measured using the HEMOCLOT thrombin inhibitor assay (HYPHEN BioMed, Neuville sur Oise, France) according to the manufacturer's instructions. This assay is used for the quantitative measurement of dabigatran in plasma (within the range $50-500 \mathrm{ng} / \mathrm{mL}$ ), with a clotting method based on the inhibition of a constant and defined concentration of thrombin. Briefly, diluted test plasma (50 $\mu \mathrm{L}$ plasma sample $+350 \mu \mathrm{L} 0.9 \% \mathrm{NaCl}$ ) was mixed with the normal pooled human plasma provided in the HEMOCLOT assay kit (reagent R1) and incubated $\left(2 \mathrm{~min}\right.$ at $\left.37^{\circ} \mathrm{C}\right)$. Clotting is initiated by adding a constant amount of highly purified human $\alpha$-thrombin (reagent R2), and the time lag between the addition of R2 and the clot formation is detected in seconds as clotting time and used in the final presentation of the data.

To measure aPTT, we added a kaolin-cephalin reagent, which is a mixture of cephalin and microcrystalline kieselguhr $(75 \mu \mathrm{L})$, to the plasma sample $(75 \mu \mathrm{L})$ and incubated the mixture at $37^{\circ} \mathrm{C}$ for 3 min under continuous stirring. Subsequent addition of $\mathrm{CaCl}_{2}$ solution $(0.025 \mathrm{M})$ activated the endogenic clotting system, leading to fibrin formation. 
The time from the addition of $\mathrm{CaCl}_{2}$ to the formation of a fibrin clot was measured as aPTT and used in the final presentation of the data. The reagent kit was from Roche Diagnostics GmbH (Mannheim, Germany).

The ECT assay is based on the properties of ecarin, a metalloprotease isolated from the venom of the saw-scaled viper Echis carinatus, which generates meizothrombin from prothrombin by specific cleavage of prothrombin at Arg 320. The procoagulant activity of meizothrombin is low, amounting to approximately $10 \%$ of the equimolar thrombin mass, making the assay less sensitive than a direct assay of thrombin activity. The assay procedure was as follows: a plasma sample $(75 \mu \mathrm{L})$ was added to imidazole/Veronal buffer $(75 \mu \mathrm{L})$ and incubated for 2 min at $37^{\circ} \mathrm{C}$. The measurement is initiated by the addition of $75 \mu \mathrm{L}(6 \mathrm{U} / \mathrm{mL})$ ecarin solution (Pentapharm), leading to conversion of prothrombin into thrombin. The time lag between the addition of ecarin and the clot formation is detected as ECT and used in the final presentation of the data.

\section{Safety}

The evaluation of safety was based on the incidence of adverse events (AEs), physical examinations, measurement of vital signs (blood pressure, pulse rate), 12-lead ECGs, clinical laboratory tests (hematology, clinical chemistry, and urinalysis), local tolerability, and a global assessment of tolerability by the investigator.

\section{Statistical methods}

Point estimators [geometric means (gMean)] of the median intra-subject ratios (with/without enoxaparin pre-treatment) of $\mathrm{AUC}_{0-\infty}, \mathrm{C}_{\max }, \mathrm{AUEC}_{0-48}$, and $\mathrm{ER}_{\max }$, and their twosided $90 \%$ confidence intervals (CIs) were calculated. This study was designed as a relative bioavailability study in a cross-over fashion, which is considered to be the most appropriate design to investigate drug interactions. The statistical model was an analysis of variance (ANOVA) on logtransformed parameters, including effects for "sequence", "subjects nested within sequences", "period", and "treatment". CIs were based on the residual error from the ANOVA and were used to estimate the probability of interaction between the two drugs. For all other parameters, descriptive statistics were calculated.

\section{Results}

Subjects

A total of 24 subjects entered the trial (11 women, 13 men), but one male subject was withdrawn before administration of the first dose due to an AE. PK, PD, and safety data were available for 23 subjects during each treatment sequence. Overall, the mean ( \pm standard deviation) age of the 23 subjects was $38.4 \pm 8.5$ years, and the BMI was $23.38 \pm$ $2.23 \mathrm{~kg} / \mathrm{m}^{2}$.

\section{PK parameters}

The administration of the last dose of enoxaparin $24 \mathrm{~h}$ prior to dabigatran etexilate $220 \mathrm{mg}$ did not markedly affect the plasma concentration-time profile of total dabigatran (Fig. 1). Similar profiles were also observed for plasma concentrations of free dabigatran.

There was no clinically relevant effect of enoxaparin pretreatment on the PK of total or free dabigatran (Table 1). The exposure to dabigatran after the switch from enoxaparin was slightly lower compared to the reference treatment without enoxaparin pre-treatment (Table 1). The test/reference ratio of $\mathrm{AUC}_{0-\infty}$ for total dabigatran was $84 \%(90 \%$ CI $67.2-$ $105.0)$ and that for $C_{\max }$ was $86 \%(90 \%$ CI $67.0-110.0)$, representing an average decrease of approximately $15 \%$ in the bioavailability of dabigatran $24 \mathrm{~h}$ after pre-treatment with enoxaparin. The values for time from dosing to maximum plasma concentration and terminal half-life were similar for both treatment periods. The ratio of free to total dabigatran gMean $\mathrm{AUC}_{0-\infty}$ was not changed by the enoxaparin pre-treatment (dabigatran alone 0.760 ; after enoxaparin 0.765).

PD parameters

The effect-time profiles for PiCT, aPTT, ECT, and dTT are shown in Fig. 2. When dabigatran etexilate was dosed $24 \mathrm{~h}$ after enoxaparin, the PiCT ratio to baseline curve was more pronounced than that with the administration of dabigatran alone. The time to peak was similar $(3 \mathrm{~h})$ in both treatment regimens, but there was a slight elevation of the time to clotting at all time points in the former when compared to those obtained with dabigatran alone. This slight elevation of approximately $15 \%$ was

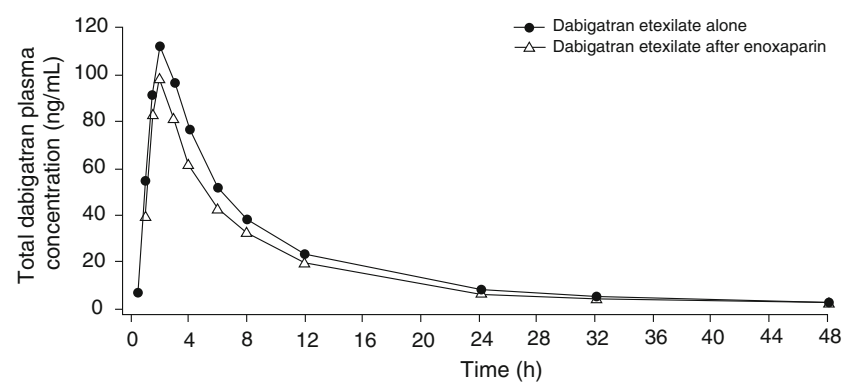

Fig. 1 Mean plasma concentration of total dabigatran following administration with and without enoxaparin 
Table 1 Adjusted by-treatment geometric means and relative bioavailability of dabigatran (total and free) after administration of dabigatran etexilate $220 \mathrm{mg}$, with and without enoxaparin $(n=23)$

\begin{tabular}{|c|c|c|c|c|c|c|c|}
\hline \multirow[t]{2}{*}{ Analyte } & \multirow[t]{2}{*}{ Parameter } & \multicolumn{2}{|l|}{ gMean } & \multirow[t]{2}{*}{$\begin{array}{l}\text { Ratio test/ } \\
\text { reference }(\%)\end{array}$} & \multicolumn{2}{|c|}{$\begin{array}{l}90 \% \text { Confidence } \\
\text { interval }\end{array}$} & \multirow[t]{2}{*}{$\begin{array}{l}\text { Intra-individual } \\
\mathrm{gCV}(\%)\end{array}$} \\
\hline & & $\begin{array}{l}\text { Test }^{\mathrm{a}} \\
(n=23)\end{array}$ & $\begin{array}{l}\text { Reference }^{\mathrm{b}} \\
(n=23)\end{array}$ & & $\begin{array}{l}\text { Lower } \\
\text { limit }\end{array}$ & $\begin{array}{l}\text { Upper } \\
\text { limit }\end{array}$ & \\
\hline \multirow[t]{2}{*}{ Total dabigatran } & $\operatorname{AUC}_{0-\infty}(\mathrm{ng} \cdot \mathrm{h} / \mathrm{mL})$ & 774.2 & 921.7 & 84 & 67.2 & 105.0 & 46.1 \\
\hline & $\mathrm{C}_{\max }(\mathrm{ng} / \mathrm{mL})$ & 99.0 & 115.3 & 86 & 67.0 & 110.0 & 51.9 \\
\hline \multirow[t]{2}{*}{ Free dabigatran } & $\operatorname{AUC}_{0-\infty}(\mathrm{ng} \cdot \mathrm{h} / \mathrm{mL})$ & 591.7 & 700.1 & 85 & 67.5 & 105.9 & 46.6 \\
\hline & $\mathrm{C}_{\max }(\mathrm{ng} / \mathrm{mL})$ & 78.4 & 90.7 & 86 & 67.5 & 110.8 & 51.9 \\
\hline
\end{tabular}

gMean, Geometric mean; $\mathrm{AUC}_{0-\infty}$, area under the concentration-time curve; $\mathrm{C}_{\max }$, maximum plasma concentration; gCV, geometric coefficient of variation

a Test: dabigatran etexilate after 3-day subcutaneous treatment with enoxaparin

${ }^{\mathrm{b}}$ Reference: dabigatran etexilate alone

relatively consistent over time. Using the primary endpoints measured over the entire 48-h time period, the test/reference ratio for $\mathrm{AUEC}_{0-48}$ was $163.7 \%(90 \% \mathrm{CI}$ 114.3-234.3), an increase of approximately $64 \%$, and the ratio for $\mathrm{ER}_{\max }$ was $115.2 \%(90 \%$ CI $101.8-130.4)$, an increase of approximately $15 \%$ compared with dabigatran etexilate alone. Mean PiCT levels were higher in female than in male subjects (data not shown).

The dTT and ECT only measure the specific effects of antithrombin activity - the former by adding exogenous thrombin to diluted plasma and the latter by activating endogenous thrombin, thus bypassing any effect of factor $\mathrm{Xa}$ inhibition. These coagulation parameters were not meaningfully affected by enoxaparin (Fig. 2).

The aPTT assay is a performance indicator of the efficacy of the intrinsic pathway of the coagulation cascade, involving factors I, II, V, VIII, IX X, XI, and XII. Enoxaparin pretreatment increased the $\mathrm{AUEC}_{0-48}$ of aPTT by approximately $14 \%$ (Table 2), and except for a slight discrepancy in anticoagulation at $12 \mathrm{~h}$, the aPTT ratio curves for dabigatran alone and dabigatran after enoxaparin were almost superimposable (Fig. 2).

\section{PK/PD correlation}

Evaluation of the PK/PD revealed a curvilinear relationship between plasma concentration and coagulation prolongation for aPTT (Fig. 3a). No effect of enoxaparin on the PK/PD relationship of aPTT was found when dabigatran etexilate was given alone or in combination with enoxaparin. The PiCT assay also exhibited a curvilinear relationship with plasma concentrations of dabigatran (Fig. 3b). A slight shift of the curve was observed when dabigatran etexilate was administered after enoxaparin, due the slight elevation of time to clotting observed with this assay (Fig. 1).
Safety

The occurrence of AEs is shown in Table 3. Eight subjects reported a total of $12 \mathrm{AEs}$, two of which were considered treatment-related. The AEs reported during treatment were headache ( 3 subjects), fatigue ( 2 subjects), back pain (2 subjects), diarrhea, hematoma, epistaxis, rash, and increase in hepatic enzymes (1 subject each). Of these, all but one AE were of mild nature. The increase in hepatic enzymes during treatment with enoxaparin + dabigatran in one subject was considered moderate. This patient already had a slight elevation at baseline; on re-testing after 10 days the levels had returned to baseline. There were no serious AEs or AEs of severe intensity during treatment.

The actual treatment at onset of the AEs was dabigatran in three subjects $(13.0 \%)$, enoxaparin in four subjects $(17.4 \%)$, and enoxaparin + dabigatran in three subjects (13.0\%). One subject experienced a serious AE during the post-study period, approximately 7 weeks after the last dose. The event (ischemic stroke) required hospitalization but was not considered to be related to the study treatments. There were no clinically relevant differences in other safety parameters. Tolerability was described as "good" for both treatments.

\section{Discussion}

Guidelines for VTE prevention currently recommend therapy with a low-molecular-weight heparin (LMWH), fondaparinux, or low-dose unfractionated heparin. For high-risk patients, such as those undergoing total hip or knee arthroplasty, pharmacological thromboprophylaxis should be continued for at least 10 - and up to 35-days [1-4]. For acute treatment of VTE, unfractionated heparin, LMWH, or 
Fig. 2 Arithmetic mean effecttime profiles for prothrombinaseinduced clotting time (PiCT; a), activated partial thromboplastin time $(a P T T ; \mathbf{b})$, ecarin clotting time $(E C T ; \mathbf{c})$, and diluted thrombin time ( $d T T ; \mathbf{d}) \cdot y$-axes are ratios to baseline. Only the negative standard deviation (SD) is displayed for dabigatran etexilate alone data and only the positive SD is displayed for data from dabigatran etexilate after the enoxaparin treatment arm a

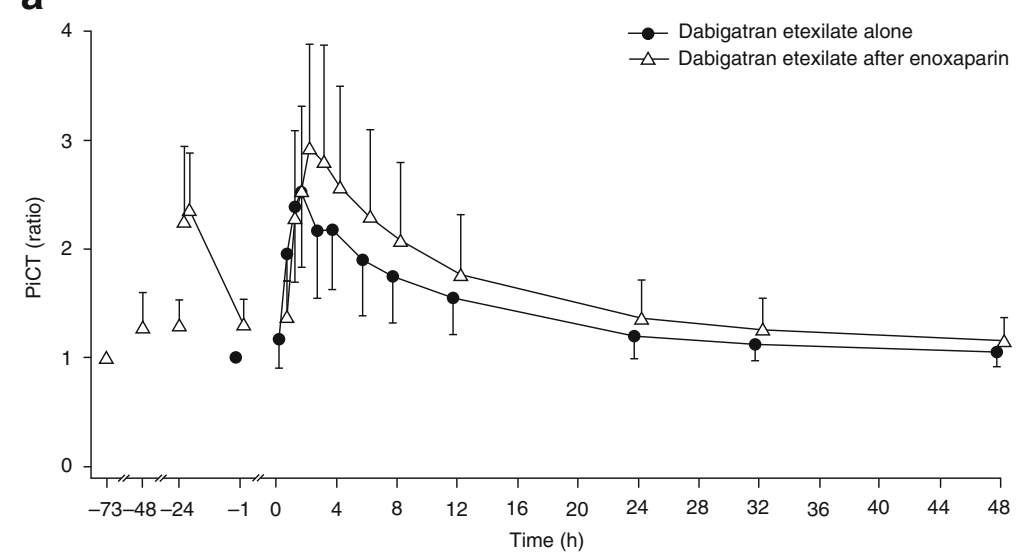

b

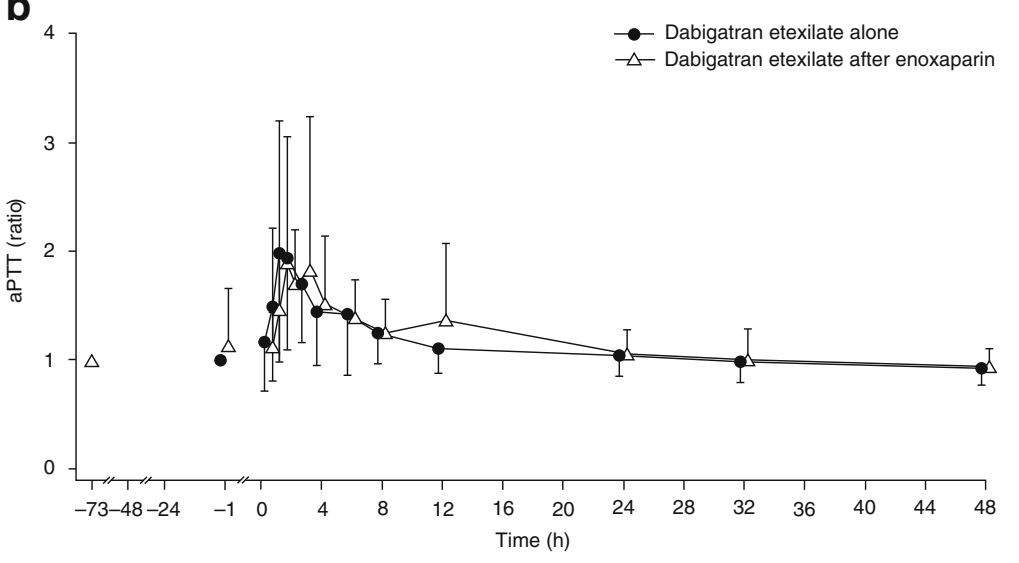

C

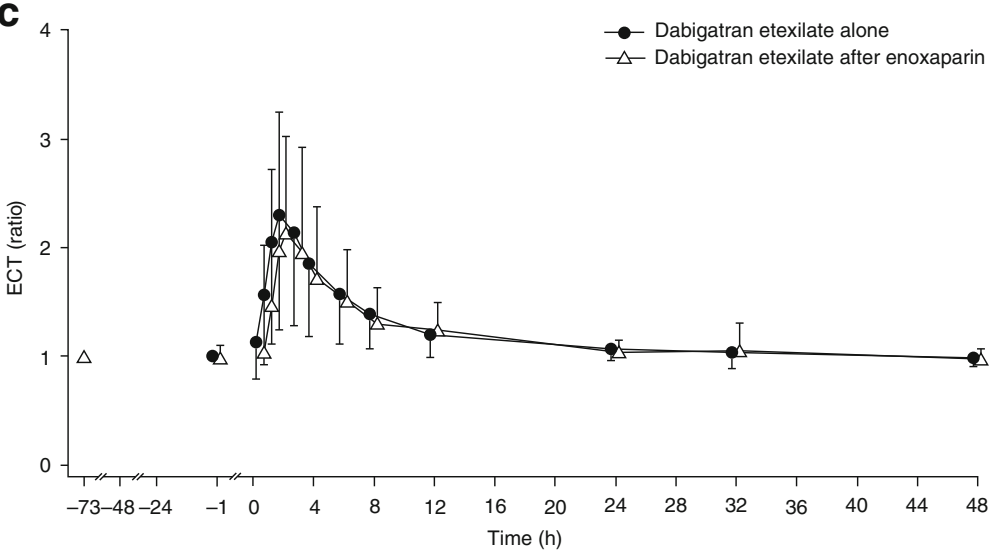

d

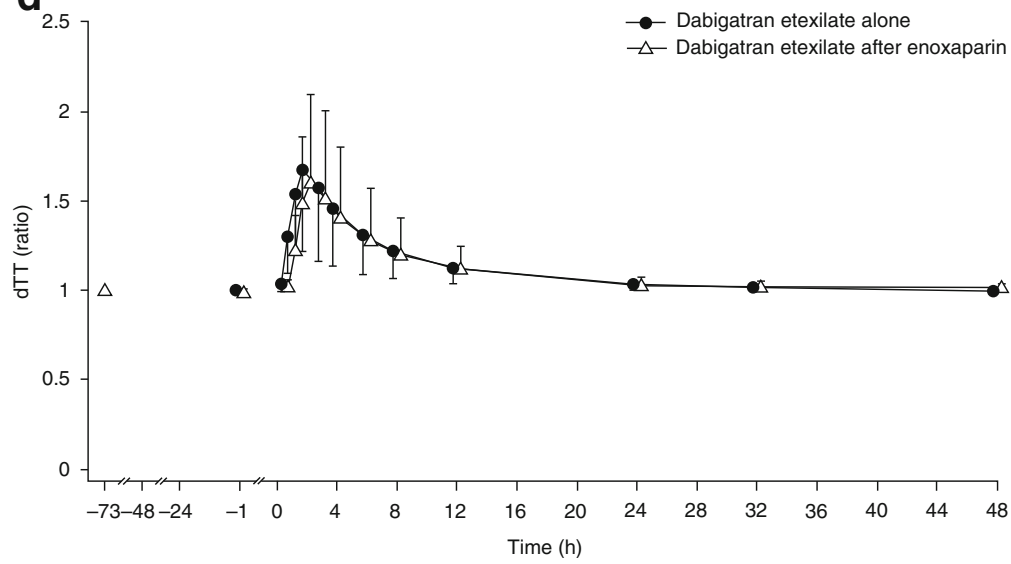


Table 2 Pharmacodynamic parameters after the administration of dabigatran etexilate $220 \mathrm{mg}$, with and without enoxaparin $(n=23)^{\mathrm{a}}$

\begin{tabular}{|c|c|c|c|c|c|}
\hline \multirow[t]{2}{*}{ Pharmacodynamic parameters } & \multicolumn{2}{|c|}{ Dabigatran etexilate alone } & \multicolumn{2}{|c|}{$\begin{array}{l}\text { Dabigatran etexilate } \\
\text { after enoxaparin }\end{array}$} & \multirow[t]{2}{*}{$\begin{array}{l}\text { Ratio of adjusted test/reference } \\
\text { [ gMean, \% }(90 \% \text { CI })]\end{array}$} \\
\hline & gMean & $\mathrm{gCV}(\%)$ & gMean & $\mathrm{gCV}(\%)$ & \\
\hline \multicolumn{6}{|l|}{ PiCT } \\
\hline $\mathrm{E}_{\text {base }}(\mathrm{s})$ & 28.4 & 18.7 & $34.7^{\mathrm{c}}$ & 10.6 & \\
\hline $\mathrm{AUEC}_{0-48}(\mathrm{~h})$ & 14.6 & 112 & $23.8^{\mathrm{c}}$ & 60.4 & $163.7(114.3-234.3)$ \\
\hline $\mathrm{ER}_{\max }$ & 2.53 & 33.0 & $2.92^{\mathrm{c}}$ & 30.0 & $115.2(101.8-130.4)$ \\
\hline \multicolumn{6}{|l|}{ aPTT } \\
\hline $\mathrm{E}_{\text {base }}(\mathrm{s})$ & 39.1 & 26.1 & 39.3 & 30.9 & \\
\hline $\mathrm{AUEC}_{0-48}(\mathrm{~h})$ & 5.65 & 106 & 6.56 & 131 & $114.2(75.1-173.5)$ \\
\hline $\mathrm{ER}_{\max }$ & 2.40 & 47.9 & 2.07 & 47.6 & $84.9(70.3-102.5)$ \\
\hline \multicolumn{6}{|l|}{ ECT } \\
\hline $\mathrm{E}_{\text {base }}(\mathrm{s})$ & 33.1 & 12.8 & $32.6^{\mathrm{d}}$ & 8.96 & \\
\hline $\mathrm{AUEC}_{0-48}(\mathrm{~h})$ & 7.28 & 123 & 7.30 & 94.3 & $99.2(67.8-145.0)$ \\
\hline $\mathrm{ER}_{\max }$ & 2.25 & 37.7 & 2.05 & 38.1 & $91.0(80.0-103.5)$ \\
\hline \multicolumn{6}{|l|}{ dTT $\left(\right.$ HEMOCLOT) ${ }^{\mathrm{b}}$} \\
\hline$E_{\text {base }}(s)$ & 31.7 & 1.67 & 31.0 & 3.24 & \\
\hline $\mathrm{AUEC}_{0-48}(\mathrm{~h})$ & 4.01 & 84.2 & 3.61 & 99.5 & $88.4(66.8-116.9)$ \\
\hline $\mathrm{ER}_{\max }$ & 1.64 & 26.6 & 1.55 & 28.2 & $95.0(87.4-103.2)$ \\
\hline
\end{tabular}

PiCT, Prothrombinase-induced clotting time; $\mathrm{E}_{\text {base }}$, baseline effect; $\mathrm{AUEC}_{0-48}$, area under the effect-time curve from 0 to $48 \mathrm{~h}$ after administration; $\mathrm{ER}_{\max }$, maximum effect ratio to baseline; aPTT, activated partial thromboplastin time; ECT, ecarin clotting time; for other pharmacodynamic parameters, see footnote of Table 1

${ }^{a}$ All of the PD assays have previously been calibrated with dabigatran standards at concentrations within the range $0.47-472 \mathrm{ng} / \mathrm{mL}$, and therefore within the expected dabigatran plasma levels [14]. For the purposes of this study, one standard was prepared at a concentration of $142 \mathrm{ng} / \mathrm{mL}$; this quality control sample was measured at the beginning, middle, and end of the test phase of each day.

${ }^{\mathrm{b}}$ dTT, Diluted thrombin time, measured using the HEMOCLOT thrombin inhibitor assay (HYPHEN BioMed, Neuville sur Oise, France)

${ }^{\mathrm{c}} n=21$;

${ }^{\mathrm{d}}{ }_{n=22}$

fondaparinux is recommended for at least 5 days; an oral vitamin $\mathrm{K}$ antagonist should be initiated in parallel, and the injectable therapy can be stopped when the international normalized ratio (INR) has reached $\geq 2$ for at least $24 \mathrm{~h}$. Oral therapy is then continued for months to years, according to patient risk factors, to prevent secondary recurrence [3-6]. Thus, when longer term anticoagulant therapy is indicated, or when treatment is to continue out of hospital for more than a few weeks, patients are usually switched from a parenteral to an oral anticoagulant due to the inconvenience of parenteral administration [3-6].

Until recently, warfarin or other vitamin $\mathrm{K}$ antagonists were the only available oral anticoagulants. However, their use is limited by slow onset of action, variability of effect, frequent INR testing, and need for dosage adjustment. As a result, therapy is not always provided or continued for the recommended duration, putting patients at risk.

This study addressed the feasibility of switching from the LMWH enoxaparin to oral therapy with the direct thrombin inhibitor dabigatran etexilate. The doses and administration schedules selected for this trial reflect the European standard clinical doses for primary VTE prevention in the orthopedic setting. Enoxaparin is generally administered at a daily dose of 20 or $40 \mathrm{mg}$ s.c. [15]. The dabigatran etexilate dose administered in this trial ( $220 \mathrm{mg}$ as a single oral dose) is the higher of two once-daily doses approved by the European Commission for the prevention of VTE in patients undergoing hip or knee surgery.

In the current study, 3-day treatment with enoxaparin $40 \mathrm{mg}$ once daily followed $24 \mathrm{~h}$ later with dabigatran etexilate resulted in small reductions in dabigatran exposure $\left(\mathrm{AUC}_{0-\infty}\right.$ and $\mathrm{C}_{\max }$ ). However, the $90 \% \mathrm{CI}$ around the intra-subject test/reference ratios included the value $100 \%$, and the ratios of free versus total dabigatran were not changed by pre-treatment with enoxaparin. After a single dose of dabigatran etexilate, the intra-subject variability was relatively high (in this study $>40 \%$ for $A U C$ and $C_{\max }$ ) and, due to the study sample size, it was not expected to be possible to demonstrate equivalence of exposure between the drug administered alone or after enoxaparin. The slight reduction in dabigatran exposure by approximately $15 \%$, however, provides evidence that pre-treatment with 
Fig. 3 Pharmacokinetic/ pharmacodynamic correlation for activated partial thromboplastin time (aPTT; a) and prothrombinase-induced clotting time (PiCT; b)

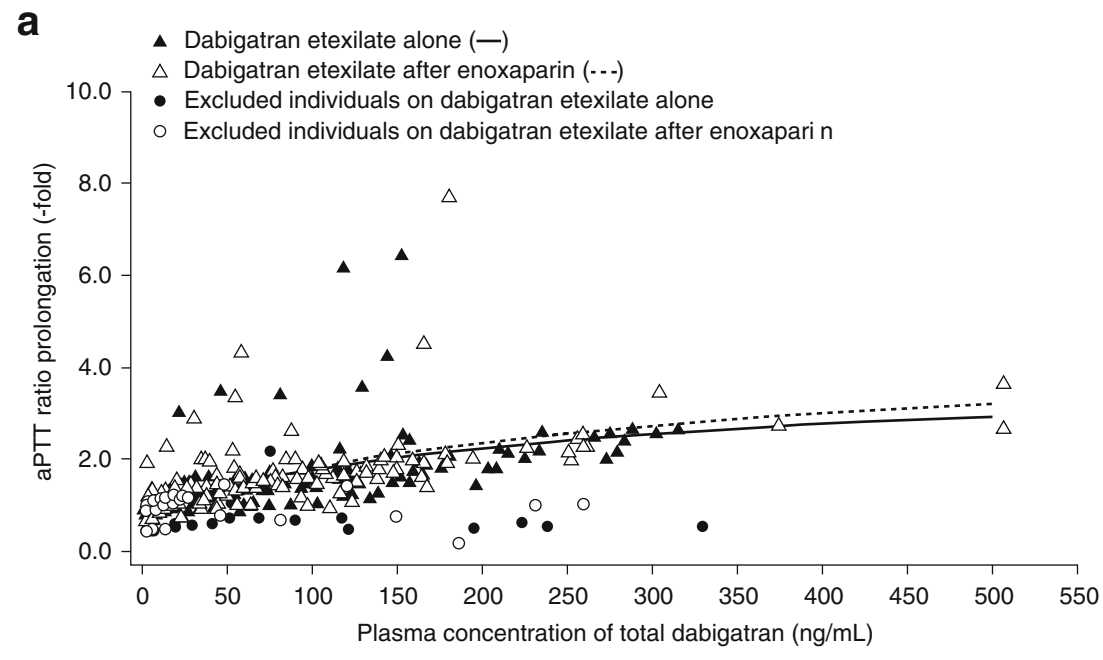

b

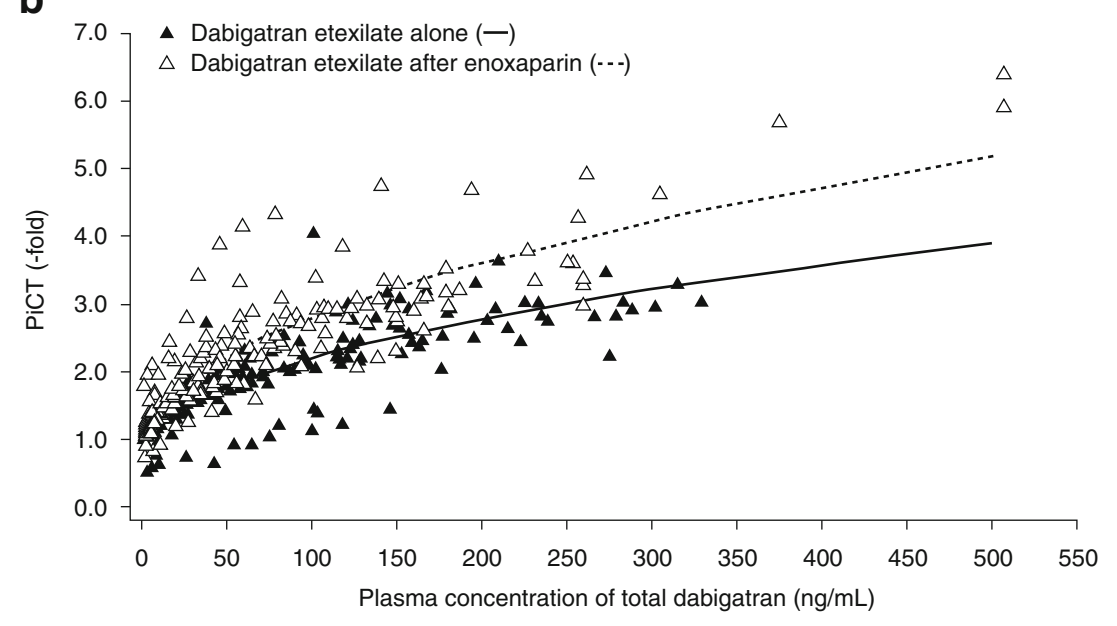

enoxaparin has no clinically meaningful effect on the PK of dabigatran.

Prolongation in the PiCT assay can result from both factor Xa inhibition and thrombin inhibition, since coagulation is preactivated in the prothrombinase complex by direct factor $\mathrm{V}$ activation. Thus, this assay has the potential to reflect the anticoagulant effect of both enoxaparin and dabigatran. There was an approximate $15 \%$ elevation of anticoagulant activity $\left(\mathrm{ER}_{\max }\right)$ in the subjects receiving dabigatran $24 \mathrm{~h}$ after enoxaparin as compared with those receiving dabigatran alone. This elevation most likely demonstrates the additive effect of dabigatran anticoagulant activity and residual anticoagulant activity from enoxaparin. The relatively large calculated primary endpoint of $64 \%$ elevation of PiCT AUEC $_{0-48}$ with enoxaparin pre-dosing also reflects the very long measurement period of $48 \mathrm{~h}$ and amplifies even very small differences between the two curves. The approximate $15 \%$ increase in $\mathrm{ER}_{\max }$ probably more appropriately reflects the real remaining effect of enoxaparin, and this elevation is not considered clinically relevant (Fig. 2).
It is also worth considering that the PiCT assay may not be the most appropriate assay for dabigatran quantitation.

Table 3 Number of adverse events reported

\begin{tabular}{llll}
\hline $\begin{array}{l}\text { Adverse } \\
\text { events }\end{array}$ & \multicolumn{2}{l}{ Treatment at onset $(n=23)$} & \\
\cline { 2 - 4 } & $\begin{array}{l}\text { Dabigatran } \\
\text { etexilate alone }\end{array}$ & Enoxaparin & $\begin{array}{l}\text { Dabigatran etexilate } \\
\text { after enoxaparin }\end{array}$ \\
\hline Headache & 1 & 2 & - \\
Diarrhea & 1 & - & - \\
Back pain & 1 & - & 1 \\
Hematoma & - & 1 & - \\
Epistaxis & - & $1^{\text {a }}$ & - \\
Fatigue & - & 2 & - \\
Rash & - & - & 1 \\
Elevated liver & & - & $1^{\text {a }}$ \\
enzymes & & & \\
\hline
\end{tabular}

${ }^{\mathrm{a}}$ Considered to be drug-related 
Calibration curves constructed for dabigatran (data not shown) highlighted a non-linear quantitation with poor precision and accuracy. Enoxaparin calibration curves, by contrast, showed a linear relation between PiCT values and enoxaparin concentration with good accuracy (data not shown).

By contrast, assays that focus predominantly on antithrombin activity, such as the dTT, which adds exogenous thrombin to diluted plasma, or the ECT, which activates prothrombin independently of the prothrombin complex, showed no difference in the anticoagulant effect with or without prior enoxaparin treatment. These data are also consistent with the plasma level results measured with LC-MS/ MS. Although enoxaparin also has anti-thrombin activity

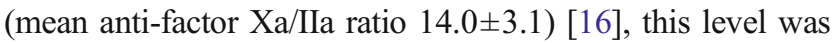
either too low to be detectable or these clotting assays are not sensitive enough to detect it. Furthermore, the relative contribution of enoxaparin's anti-thrombin activity is too small to be relevant $24 \mathrm{~h}$ after enoxaparin has been administered. This is consistent with the mechanism of action of dabigatran and correlates well with the results obtained.

No drug-related AEs, such as any bleeding or hematoma, were observed during the treatment with dabigatran etexilate after enoxaparin. Overall, a moderate increase in PiCT $24 \mathrm{~h}$ after the switch from enoxaparin to dabigatran, as observed in this trial, is not considered to be clinically meaningful.

Additionally, supportive clinical experience regarding switching was observed in another clinical setting, the treatment of VTE. In the phase III RE-COVER study, all subjects received a parenteral anticoagulant (mostly LMWH, which would typically be given at a higher dose than for primary VTE prevention) as well as either warfarin or warfarin placebo for at least 5 days (median 9 days) prior to discontinuing the parenteral anticoagulant and either switching to dabigatran etexilate $150 \mathrm{mg}$ twice daily or continuing warfarin in a double-blind, double-dummy fashion. The first dabigatran etexilate dose was given $0-2 \mathrm{~h}$ before the time when the next dose of the parenteral therapy would have been due. In this trial, there were fewer major or clinically relevant non-major bleeding events and similar rates of major bleeding events for the dabigatran versus the warfarin group during the 6-month treatment period [17, 18]. This study highlights the efficacy and safety of switching to dabigatran etexilate in this patient population, even after $12 \mathrm{~h}$ of the last dose of enoxaparin. Furthermore, in patients with atrial fibrillation, the impact of temporary treatment interruptions for surgery or a diagnostic procedure was investigated in the RE-LY trial of dabigatran etexilate 150 or $110 \mathrm{mg}$ twice daily (double blind) versus warfarin (open label) for stroke prevention in patients with atrial fibrillation $[9,10]$. Discontinuation of dabigatran etexilate for between 1 and 5 days was recommended depending on the type of procedure planned and the anticipated bleeding risk, and short-term bridging anticoagulant therapy (e.g., unfractionated heparin and/or LMWH) was used, if appropriate. Temporary interruptions occurred in 4,623 subjects. More subjects in the dabigatran etexilate groups were managed without bridging therapy ( $77.4 \%$ in the $150 \mathrm{mg}$ twice-daily group; $79.3 \%$ in the $110 \mathrm{mg}$ twice-daily group) than in the warfarin group (65.7\%). Stroke, myocardial infarction, and bleeding events occurred at similar frequencies in all treatment groups within 30 days of reinitiating study medication [18]. Thus, recommendations for switching between dabigatran etexilate and a parenteral anticoagulant resulted in effective and safe management of patients.

Similarly, in the SPORTIF III and V trials [19, 20], two Phase III trials for the prevention of stroke and systemic embolism in patients with atrial fibrillation, patients were randomized to either warfarin or ximelagatran, another direct thrombin inhibitor. As in the previously described RELY trial, there was a proportion of participants undergoing interventions during the study who were switched from enoxaparin to ximelagatran. No safety issue has been reported in relation to switching from parenteral to oral anticoagulation in these trials. In another trial of VTE treatment and secondary prevention, the THRIVE III study, switching from 6 months of standard parenteral coagulation therapy to ximelagatran for an additional 18 months effectively prevented recurrences of VTE, with an incidence of bleeding similar to that in the placebo group and low overall mortality [21]. Switching between parenteral and oral anticoagulant therapy was therefore an effective treatment option. Finally, a clinical trial investigating the effects of switching from enoxaparin to the new oral factor Xa inhibitor, rivaroxaban, in patients undergoing total hip or knee replacement has been completed; however to the best of our knowledge, results have not yet been published [22].

In summary, in this Phase I study, enoxaparin and dabigatran etexilate had favorable safety and were well tolerated; the switch from enoxaparin to dabigatran etexilate was also well tolerated. These data support the feasibility and safety of switching from enoxaparin to dabigatran etexilate.

Acknowledgments This study was supported by Boehringer Ingelheim Pharma GmbH. Writing and editorial assistance was provided by PAREXEL, which was contracted by Boehringer Ingelheim for these services.

Conflicts of interest All authors are employees of Boehringer Ingelheim.

Open Access This article is distributed under the terms of the Creative Commons Attribution Noncommercial License which permits any noncommercial use, distribution, and reproduction in any medium, provided the original author(s) and source are credited. 


\section{References}

1. Geerts WH, Bergqvist D, Pineo GF, Heit JA, Samama CM, Lassen MR, Colwell CW, American College of Chest Physicians (2008) Prevention of venous thromboembolism: American College of Chest Physicians evidence-based clinical practice guidelines (8th edition). Chest 133:429S-433S

2. Randelli F, Biggi F, Della Rocca G, Grossi P, Imberti D, Landolfi R, Palareti G, Prisco D (2011) Italian intersociety consensus statement on antithrombotic prophylaxis in hip and knee replacement and in femoral neck fracture surgery. J Orthop Traumatol 12:69-76

3. Scottish Intercollegiate Guidelines Network (SIGN) (2010) Prevention and management of venous thromboembolism. A national clinical guideline. Available at: http://www.sign.ac.uk/pdf/ sign122.pdf

4. Cardiovascular Disease Educational and Research Trust; Cyprus Cardiovascular Disease Educational and Research Trust; European Venous Forum; International Surgical Thrombosis Forum; International Union of Angiology; Union Internationale de Phlébologie (2006) Prevention and treatment of venous thromboembolism. International Consensus Statement (guidelines according to scientific evidence). Int Angiol 25:101-161

5. Kearon C, Kahn SR, Agnelli A, Raskob GE, Comerota AJ, American College of Chest Physicians (2008) Antithrombotic therapy for venous thromboembolic disease: American College of Chest Physicians evidence-based Clinical practice guidelines (8th edition). Chest 133:454S-545S

6. Torbicki A, Perrier A, Konstantinides S, Agnelli G, Galiè N, Pruszczyk P, Bengel F, Brady AJ, Ferreira D, Janssens U, Klepetko W, Mayer E, Remy-Jardin M, Bassand JP, ESC Committee for Practice Guidelines (CPG) (2008) Guidelines on the diagnosis and management of acute pulmonary embolism: the Task Force for the Diagnosis and Management of Acute Pulmonary Embolism of the European Society of Cardiology (ESC). Eur Heart J 29:2276-2315

7. European Medicines Agency (2011) Pradaxa summary of product characteristics. Available at: http://www.ema.europa.eu/docs/ en_GB/document_library/EPAR_-_Product_Information/human/ 000829/WC500041059.pdf. Accessed 3 June 2011

8. Boehringer Ingelheim (2010) Pradaxa monograph. Available at: http://www.boehringer-ingelheim.ca/en/Home/Human_Health/ Our_Products/Product_Monographs/Pradax-pm.pdf . Accessed 11 Mar 2011

9. Connolly SJ, Ezekowitz MD, Yusuf S, Eikelboom J, Oldgren J, Parekh A, Pogue J, Reilly PA, Themeles E, Varrone J, Wang S, Alings M, Xavier D, Zhu J, Diaz R, Lewis BS, Darius H, Diener HC, Joyner CD, Wallentin L (2009) Dabigatran versus warfarin in patients with atrial fibrillation. N Engl J Med 361:1139-1151

10. Connolly SJ, Ezekowitz MD, Yusuf S, Reilly PA, Wallentin L; Randomized Evaluation of Long-Term Anticoagulation Therapy Investigators (2010) Newly identified events in the RE-LY trial. N Engl J Med 363:1875-1876
11. Federal Drugs Administration (2010) Pradaxa highlights of prescribing information. Available at: http://www.accessdata.fda.gov/ drugsatfda_docs/label/2010/022512s000lbl.pdf. Accessed 06 Apr 2011

12. Pharma Times Online (2011) Europe OK for Boehringer's Pradaxa to prevent AF-related strokes. Available at: http:// www.pharmatimes.com/article/11-08-05/Europe_OK_for Boehringer_s_Pradaxa_to_prevent_AF-related_strokes.aspx. Accessed 11 Aug 2011

13. Blech S, Ebner T, Ludwig-Schwellinger E, Stangier J, Roth W (2008) The metabolism and disposition of the oral direct thrombin inhibitor, dabigatran, in humans. Drug Metab Dispos 36:386-399

14. van Ryn J, Stangier J, Haertter S, Liesenfeld KH, Wienen W, Feuring M, Clemens A (2010) Dabigatran etexilate - a novel, reversible, oral direct thrombin inhibitor: interpretation of coagulation assays and reversal of anticoagulant activity. Thromb Haemost 103:1116-1127

15. Sanofi-Aventis (2011) Clexane summary of product characteristics. Available at: http://www.sanofi.co.uk/products/Clexane_SPC. pdf. Accessed 11 Aug 2011

16. Sanofi-Aventis (2011) Lovenox (enoxaparin sodium injection) for subcutaneous and intravenous use. Prescribing information. Available at: http://products.sanofi.us/lovenox/lovenox.html. Accessed 22 Nov 2011

17. RE-COVER Study Group, Schulman S, Kearon C, Kakkar AK, Mismetti P, Schellong S, Eriksson H, Baanstra D, Schnee J, Goldhaber SZ (2009) Dabigatran versus warfarin in the treatment of acute venous thromboembolism. N Engl J Med 361:2342-2352

18. Boehringer Ingelheim (2010) Advisory committee briefing document on dabigatran etexilate. Available at: http://www.fda.gov/ downloads/AdvisoryCommittees/CommitteesMeetingMaterials/ Drugs/CardiovascularandRenalDrugsAdvisoryCommittee/ UCM226009.pdf Accessed 29 July 2011

19. Olsson SB, Executive Steering Committee of the SPORTIF III Investigators (2003) Stroke prevention with the oral direct thrombin inhibitor ximelagatran compared with warfarin in patients with non-valvular atrial fibrillation (SPORTIF III): randomised controlled trial. Lancet 362:1691-1698

20. Albers GW, Diener HC, Frison L, Grind M, Nevinson M, Partridge S, Halperin JL, Horrow J, Olsson SB, Petersen P, Vahanian A, SPORTIF Executive Steering Committee for the SPORTIF V Investigators (2005) Ximelagatran vs warfarin for stroke prevention in patients with nonvalvular atrial fibrillation: a randomized trial. JAMA 293:690-698

21. Schulman S, Wåhlander K, Lundström T, Clason SB, Eriksson H; Investigators THRIVEIII (2003) Secondary prevention of venous thromboembolism with the oral direct thrombin inhibitor ximelagatran. N Engl J Med 349:1713-1721

22. U.S. National Institute of Health (Clinical Trials.Gov) (2011) Switching drug therapy for the prevention of blood clot formation from enoxaparin to rivaroxaban after orthopedic surgery for either total hip or total knee replacement. Available at:http://clinicaltrials. gov/ct2/show/study/NCT01094886 Accessed 1 Dec 2011 